\title{
Identification of Rice Genotypes for Resistance against Yellow Stem Borer in Irrigated Rice
}

\author{
D. Sudha Rani ${ }^{1^{*}}$, Ch. Chiranjeevi ${ }^{2}$, T. Madhumathi ${ }^{2}$, \\ S. Krishnam Raju ${ }^{3}$ and Sk. Nafeez Umar ${ }^{4}$ \\ ${ }^{1}$ Entomology, Agricultural Research Station, Garikapadu, Krishna District, \\ Andhra Pradesh, India \\ ${ }^{2}$ Department of Entomology, ${ }^{4}$ Department of Statistics and Mathematics, Agricultural \\ College, Bapatla, Guntur district, Andhra Pradesh, India \\ ${ }^{3}$ Department of Plant Pathology, Agricultural College, Rajamahendravarum, East Godavari \\ district, Andhra Pradesh, India \\ *Corresponding author
}

\section{A B S T R A C T}

\section{Keywords}

Rice germplasm, Yellow stem borer, Augmented block design, SES scale

Article Info

Accepted:

15 April 2020

Available Online:

10 May 2020
The relative resistance or susceptibility of nearly 215 rice genotypes supplied by Indian Institute of Rice Research, Hyderabad was screened to identify the resistant genotype against rice yellow stem borer. The trial was conducted following augmented block design with two checks i.e., local check (BPT 5204) and susceptible check (TN1) for a period of two successive kharif seasons ( $2016 \&$ 2017). The per cent incidence of stem borer was recorded at their peak infestation of dead hearts at tillering stage and white ears at reproductive stage. The status of the screened genotypes was determined by following the standard evaluation scale (SES) for stem borer as suggested by IRRI. The pooled results of screening trial for two seasons inferred that, among 215 genotypes screened, no entry exhibited resistance scale, 87 entries registered moderate resistant, 116 were found moderately susceptible and 22 lines recorded the susceptible scale with respect to per cent dead hearts infestation. Correspondingly, the pooled mean data relating to per cent white ears indicated that, only 14 entries were resistant, while 101 entries had registered moderate resistant, 82 entries were moderately susceptible, 17 entries recorded susceptible status and only 1 entry had exhibited susceptible status. The rice entries which had exhibited resistance at dead heart stage were found susceptible at white ears stage and vice versa as both the factors were independent. Hence, upon clean examination only seven rice entries with IC No. 381538, 450535, 463380, 464140, 464186, 574807 and 578388 were found to exhibit resistance or moderate resistance against yellow stem borer at both vegetative and reproductive stages of rice crop.

\section{Introduction}

In India rice crop is cultivated under wide range of altitude and climatic conditions. Rice cultivation extends from 8 to $35^{\circ} \mathrm{N}$ latitude and from sea level to as high as 3000 meters in our country. In India, for the year 2018-19 rice was cultivated in an area of 43.79 million 
hectares with 115.63 millon tones of annual production accounting around 2.64 tonnes /ha productivity (Agricultural Statistics at a glance, 2018). The major production constraint in rice cultivation includes weeds infestation, pests and diseases attack. Even though, nearly 300 insect pests known to attack rice crop 23 insect species cause remarkable damage (Pasalu and Katti, 2006). Among various pests influencing the yield of rice crop, yellow stem borer, Scirpophaga incertuals was considered as major destructive pest resulting in average yield loss of 30 per cent (Krishnaiah and Varma, 2015).

In case of severe pest incidence of stem borer especially in susceptible varieties the usage of insecticides is inevitable. Many farmers are adopting combination chemicals in order to manage the stem borers and upon regular usage of chemicals with same mode of action and in compatible insecticidal combinations may lead to pest resistance, resurgence and residual effect. Hence, practicing integrated pest management (IPM) tools against rice stem borer plays a pivotal role. The prime and major component to be adopted in IPM strategies is host plant resistance as it is compatible with other components of IPM. It is noteworthy that at present no rice germplasm had exhibited resistance against both dead hearts at vegetative stage and white ears at reproductive stage. Keeping in view the ambiguity in resistance at dead hearts and white ears stage caused by yellow stem borer in rice, a screening trial was undertaken to identify the resistance source of rice genotypes against yellow stem borer for two consecutive kharif seasons.

\section{Materials and Methods}

Screening trial was conducted following augmented block design with 215 rice germplasm sourced by Indian Institute of Rice Research (IIRR), Rajendranagar, Hyderabad at Agricultural Research Station, Garikapadu for a period of two consecutive seasons i.e., kharif, 2016 and kharif, 2017 to assess the resistance source against the rice yellow stem borer. The Augmented Block Design ABD was adopted for executing the trial in order to identify the resistant source of rice germplasm from 215 rice germplasm accessions along with susceptible check (TN1) and local check (BPT 5204) were transplanted after every fifteen entries.

Each entry was transplanted with two seedlings per hill in two rows of 20 hills with $5.0 \mathrm{~m}$ length was. The incidence of per cent dead hearts (\% DH) and per cent white ears (\%WE) were recorded on the rice entries and check varieties at the peak infestation during the vegetative stage and reproductive stage of the rice crop, respectively. The observations on $\% \mathrm{DH}$ and $\% \mathrm{WE}$ were recorded from ten randomly selected hills per entry and the per cent dead heart and white ear were calculated as per the formulae here under.

Per cent dead hearts $=$

Total number of dead hearts in 10 hills X 100

Total number of tillers in 10 hills

Per cent White ears =

$\underline{\text { Total number of white ears in } 10 \text { hills }}$ X 100

Total number of tillers in 10 hills

Based on the damage rating (per cent $\mathrm{DH}$ and per cent WE) and scale the reaction of rice genotypes towards resistance or susceptibility was determined by following the IRRI Standard Evaluation System (SES) for rice (IRRI, 2002) (Table 1 \& 2).

\section{Results and Discussion}

During kharif 2016, the peak infestation of yellow stem borer in terms of per cent dead 
hearts (\% DH) was recorded at 45 days after transplantation (DAT) whereas, during kharif, 2017 at 55 DAT peak infestation of DH were noticed. The maximum per cent white ear (\%WE) damage by yellow stem borer during reproductive stage of the rice was recorded at 125 DAT during kharif, 2016 and at 130 DAT, during kharif, 2017.

Identification of rice genotypes against yellow stem borer with respect to per cent dead hearts during vegetative stage

\section{kharif, 2016}

For the season kharif, 2016 out of 215 rice entries screened for their reaction towards resistance or susceptibility against yellow stem borer, 81 entries exhibited the scale ' 3 ' with status of moderate resistance (MR) and the per cent $\mathrm{DH}$ in these entries ranged from 10.5 (C-858) to 20.4 (C-27) . A sum of 107 entries of rice had registered moderatel susceptible (MS) reaction with per cent $\mathrm{DH}$ damage ranged between 20.5 (C-692) and 30.1 (C-1180) and rated with scale ' 5 '. A total of 27 rice entries exhibited susceptible (scale 7) reaction dead heart damage by stem borer infesting rice and the corresponding values ranged from 30.5 to 39.8 . The per cent dead hearts in check varieties was recorded as 42.2 per cent in TN1 and 32.8 per cent in BPT 5204 with scale 7 representing the susceptible status (Table 3).

\section{kharif 2017}

Among 215 rice entries screened during kharif, 2017, only one entry (C-599) registered resistance $(\mathrm{R})$ status with 10.3 per cent dead heart damage. A sum of 93 entries witnessed moderately resistant (10.6-20.4\% $\mathrm{DH})$ and 99 germplasm entries were found moderately susceptible $(20.5-30.4 \% \mathrm{DH})$ to rice yellow stem borer. A total of 22 germplasm lines were determined as susceptible (S) entries with damage greater than 31 per cent DH representing scale 7 . In check varieties the damage scale was found as ' 7 ' in TN1 (35.1 \% $\mathrm{DH})$ exhibiting susceptible (S) pest reaction and scale ' 5 ' was seen in BPT 5204 (26.9 \% DH) check with moderately susceptible (MS) pest reaction.

The summative mean of both kharif seasons data in terms of per cent dead hearts indicated that among 215 rice entries identified for reaction towards resistance or susceptibility against rice yellow stem borer, 87 entries registered moderately resistant (11-20\% DH), 106 were considered as moderately susceptible (21-30\% $\mathrm{DH})$ and 22 lines witnessed susceptible reaction with damage ranged from 31-40 per cent dead hearts DH. The lowest per cent dead hearts (11.0) were recorded in $\mathrm{C}-858$ and $\mathrm{C}-1372$ rice entries as against highest in C-358 and C-391 with 40.0 per cent DH, respectively (Table 3 ).

Identification of rice genotypes against yellow stem borer with respect to per cent white ears during reproductive stage

The rice entries (mostly) which expressed resistance $(\mathrm{R})$ or moderate resistance (MR) against dead hearts during vegetative stage were found moderately susceptible (MS) susceptible (S) to white ears damage by yellow stem borer at reproductive stage and vice versa. The white ears damage due to rice yellow stem borer incidence had ranged from 2.4 to 31.5 per cent during kharif, 2016 and 2.3 to 29.3 per cent during kharif, 2017.

\section{kharif, 2016}

During kharif, 2016215 rice entries were screened at field level to assess their reaction towards resistance or susceptibility against yellow stem borer and the results indicated that, 12 lines expressed resistance status with less than 5 per cent WE damage representing scale 1 . The lowest per cent WE damage was 
noticed in rice entry C-1433 (2.4) followed by C-1464 (3.6) rice entry as against highest incidence recorded in C-490 (31.5\% WE with scale 9 the attaining the status of highly susceptible (HS). Whereas, 12 entries registered pest status of resistance (scale 1), 95 entries were regarded as moderately resistance (scale 3), 73 were identified as moderately susceptible (scale 5), 34 were categorized as susceptible with sacle7 and only one rice entry witnessed highly susceptible pest reaction with scale 9 (Table 4).

\section{Kharif, 2017}

The resultant resistance/ susceptible reactions of 215 rice accessions field screened against white ears damage caused by yellow stem borer during kharif, 2017 revealed that 29 entries were identified as resistant (R), 105 entries were categorized moderately resistant (MR), 58 entries were regarded as moderately susceptible (MS), 22 were witnessed as susceptible (S) and only one entry exhibited highly susceptible (HS) reaction with a damage range of 2.3-5.3, 5.6-10.4, 10.5-14.8, 15.6-21.8 and $29.3 \%$ white ears, respectively. The lowest and highest per cent white ears were recorded in C-1398 and C-490 with 2.3 and 29.3 per cent, respectively. The per cent WE damage recorded in check varieties TN1 (susceptible) and BPT 5204 (susceptible/ moderately susceptible) were 17.6 and 14.6, respectively (Table 4).

Table.1 Standard Evaluation System for rice yellow stem borer with respect to per cent dead hearts

\begin{tabular}{|c|c|l|}
\hline Damage (\%) & Scale & \multicolumn{1}{|c|}{ Reaction/Status } \\
\hline $\mathbf{0}$ & 0 & Highly Resistant (HR) \\
\hline $\mathbf{1 - 1 0}$ & 1 & Resistant (R) \\
\hline $\mathbf{1 1 - 2 0}$ & 3 & Moderately Resistant (MR) \\
\hline $\mathbf{2 1 - 3 0}$ & 5 & Moderately Susceptible (MS) \\
\hline $\mathbf{3 1 - 6 0}$ & 7 & Susceptible(S) \\
\hline $\mathbf{6 1}$ \& above & 9 & Highly Susceptible(HS) \\
\hline
\end{tabular}

Table.2 Standard Evaluation System for rice yellow stem borer with respect to per cent white ears

\begin{tabular}{|c|c|l|}
\hline Damage (\%) & Scale & \multicolumn{1}{|c|}{ Reaction/ Status } \\
\hline $\mathbf{0}$ & 0 & Highly Resistant (HR) \\
\hline $\mathbf{1 - 5}$ & 1 & Resistant (R) \\
\hline $\mathbf{6 - 1 0}$ & 3 & Moderately Resistant (MR) \\
\hline $\mathbf{1 1 - 1 5}$ & 5 & Moderately Susceptible (MS) \\
\hline $\mathbf{1 6 - 2 5}$ & 7 & Susceptible(S) \\
\hline $\mathbf{2 6}$ \& above & 9 & Highly Susceptible(HS) \\
\hline
\end{tabular}


Table.3 Field identification of rice genotypes against yellow stem borer, in terms of per cent dead hearts

\begin{tabular}{|c|c|c|c|c|c|c|c|c|c|}
\hline S. No & $\begin{array}{c}\text { Entry } \\
\text { No. }\end{array}$ & $\begin{array}{c}\text { Kharif, } \\
2016\end{array}$ & Scale & Status & $\begin{array}{c}\text { Kharif } \\
2017\end{array}$ & Scale & Status & Mean & Status \\
\hline 1 & C-08 & 21.3 & 5 & MS & 28.3 & 5 & MS & 25 & MS \\
\hline 2 & C-17 & 27.6 & 5 & MS & 21.4 & 5 & MS & 25 & MS \\
\hline 3 & C-27 & 20.4 & 3 & MR & 18.3 & 3 & MR & 19 & MR \\
\hline 4 & C-30 & 20.0 & 3 & MR & 23.8 & 5 & MS & 22 & MS \\
\hline 5 & C-37 & 26.9 & 5 & MS & 27 & 5 & MS & 27 & MS \\
\hline 6 & C-53 & 18.9 & 3 & MR & 16.3 & 3 & MR & 18 & MR \\
\hline 7 & C-55 & 20.5 & 5 & MS & 22.5 & 5 & MS & 22 & MS \\
\hline 8 & C-58 & 16.9 & 3 & MR & 14.6 & 3 & MR & 16 & MR \\
\hline 9 & C-64 & 18.5 & 3 & MR & 23.4 & 5 & MS & 21 & MS \\
\hline 10 & C-86 & 22.8 & 5 & MS & 29.8 & 5 & MS & 26 & MS \\
\hline 11 & C-115 & 21.5 & 5 & MS & 31.4 & 7 & S & 26 & MS \\
\hline 12 & C-124 & 26.8 & 5 & MS & 26.9 & 5 & MS & 27 & MS \\
\hline 13 & C-133 & 21.8 & 5 & MS & 19.6 & 3 & MR & 21 & MS \\
\hline 14 & C-140 & 14.6 & 3 & MR & 13.8 & 3 & MR & 14 & MR \\
\hline 15 & C-141 & 19.8 & 3 & MR & 20.8 & 5 & MS & 20 & MR \\
\hline 16 & C-144 & 26.8 & 5 & MS & 16.3 & 3 & MR & 22 & MS \\
\hline 17 & C-152 & 21.5 & 5 & MS & 20.4 & 3 & MR & 21 & MS \\
\hline 18 & C-170 & 22.8 & 5 & MS & 28.1 & 5 & MS & 25 & MS \\
\hline 19 & C-171 & 24.5 & 5 & MS & 16.3 & 3 & MR & 20 & MR \\
\hline 20 & C-178 & 19.6 & 3 & MR & 22.4 & 5 & MS & 21 & MS \\
\hline 21 & C-202 & 13.9 & 3 & MR & 18.3 & 3 & MR & 16 & MR \\
\hline 22 & C-207 & 20.8 & 5 & MS & 22.6 & 5 & MS & 22 & MS \\
\hline 23 & $\mathrm{C}-221$ & 15.8 & 3 & MR & 18.4 & 3 & MR & 17 & MR \\
\hline 24 & C-228 & 13.8 & 3 & MR & 22.8 & 5 & MS & 18 & MR \\
\hline 25 & $\mathrm{C}-237$ & 21.6 & 5 & MS & 21.0 & 5 & MS & 21 & MS \\
\hline 26 & $\mathrm{C}-240$ & 20.5 & 5 & MS & 19.3 & 3 & MR & 20 & MR \\
\hline 27 & $\mathrm{C}-250$ & 26.8 & 5 & MS & 16.8 & 3 & MR & 22 & MS \\
\hline 28 & C-269 & 14.9 & 3 & MR & 22.8 & 5 & MS & 19 & MR \\
\hline 29 & $\mathrm{C}-270$ & 22.8 & 5 & MS & 16.9 & 3 & MR & 20 & MR \\
\hline 30 & C-273 & 20.0 & 3 & MR & 18.0 & 3 & MR & 19 & MR \\
\hline 31 & C-275 & 18.3 & 3 & MR & 22.0 & 5 & MS & 20 & MR \\
\hline 32 & C-280 & 16.8 & 3 & MR & 18.3 & 3 & MR & 18 & MR \\
\hline 33 & C-288 & 17.9 & 3 & MR & 14.3 & 3 & MR & 16 & MR \\
\hline 34 & C-306 & 18.5 & 3 & MR & 18.0 & 3 & MR & 18 & MR \\
\hline 35 & C-319 & 28.5 & 5 & MS & 20.0 & 3 & MR & 24 & MS \\
\hline 36 & C-321 & 18.8 & 3 & MR & 22.8 & 5 & MS & 21 & MS \\
\hline 37 & C-324 & 21.5 & 5 & MS & 16.5 & 3 & MR & 19 & MR \\
\hline 38 & C-328 & 26.5 & 5 & MS & 28.5 & 5 & MS & 28 & MS \\
\hline 39 & C-330 & 19.8 & 3 & MR & 16.5 & 3 & MR & 18 & MR \\
\hline 40 & C-342 & 22.8 & 5 & MS & 11.8 & 3 & MR & 17 & MR \\
\hline
\end{tabular}




\begin{tabular}{|c|c|c|c|c|c|c|c|c|c|}
\hline S. No & Entry No. & $\begin{array}{c}\text { Kharif, } \\
2016\end{array}$ & Scale & Status & $\begin{array}{c}\text { Kharif, } \\
2017\end{array}$ & Scale & Status & Mean & Status \\
\hline 41 & C-343 & 19.0 & 3 & MR & 22.6 & 5 & MS & 21 & MS \\
\hline 42 & C-346 & 16.8 & 3 & MR & 18.3 & 3 & MR & 18 & MR \\
\hline 43 & C-349 & 22.4 & 5 & MS & 16.4 & 3 & MR & 19 & MR \\
\hline 44 & C-350 & 29.3 & 5 & MS & 22.0 & 5 & MS & 26 & MS \\
\hline 45 & C-352 & 26.1 & 5 & MS & 18.6 & 3 & MR & 22 & MS \\
\hline 46 & C-354 & 28.1 & 5 & MS & 29.6 & 5 & MS & 29 & MS \\
\hline 47 & C-358 & 39.3 & 7 & $S$ & 40.1 & 7 & S & 40 & $\mathrm{~S}$ \\
\hline 48 & C-361 & 22.5 & 5 & MS & 16.9 & 3 & MR & 20 & MR \\
\hline 49 & C-362 & 18.3 & 3 & MR & 33.4 & 7 & $\mathrm{~S}$ & 26 & MS \\
\hline 50 & C-364 & 24.5 & 5 & MS & 40.8 & 7 & S & 33 & $\mathrm{~S}$ \\
\hline 51 & C-365 & 20.9 & 5 & MS & 33.1 & 7 & $S$ & 27 & MS \\
\hline 52 & C-368 & 23.8 & 5 & MS & 16.8 & 3 & MR & 20 & MR \\
\hline 53 & C-369 & 18.6 & 3 & MR & 13.2 & 3 & MR & 16 & MR \\
\hline 54 & C-372 & 13.8 & 3 & MR & 18.3 & 3 & MR & 16 & MR \\
\hline 55 & C-373 & 15.3 & 3 & MR & 14.6 & 3 & MR & 15 & MR \\
\hline 56 & C-374 & 18.3 & 3 & MR & 13.5 & 3 & MR & 16 & MR \\
\hline 57 & C-377 & 24.8 & 5 & MS & 18.4 & 3 & MR & 22 & MS \\
\hline 58 & C-378 & 23.1 & 5 & MS & 16.8 & 3 & MR & 20 & MR \\
\hline 59 & C-380 & 20.8 & 5 & MS & 13.8 & 3 & MR & 17 & MR \\
\hline 60 & C-384 & 16.8 & 3 & MR & 20.6 & 5 & MS & 19 & MR \\
\hline 61 & C-386 & 21.6 & 5 & MS & 19.8 & 3 & MR & 21 & MS \\
\hline 62 & C-388 & 20.8 & 5 & MS & 19.6 & 5 & MR & 20 & MR \\
\hline 63 & C-390 & 29.6 & 5 & MS & 34.6 & 7 & $S$ & 32 & S \\
\hline 64 & C-391 & 38.5 & 7 & $\mathrm{~S}$ & 40.6 & 7 & S & 40 & $S$ \\
\hline 65 & C-393 & 20.8 & 5 & MS & 19.3 & 3 & MR & 20 & MR \\
\hline 66 & C-394 & 36.3 & 7 & $\mathrm{~S}$ & 23.8 & 5 & MS & 30 & MS \\
\hline 67 & C-396 & 28.2 & 5 & MS & 10.8 & 3 & MR & 20 & MR \\
\hline 68 & C-400 & 16.5 & 3 & MR & 22.6 & 5 & MS & 20 & MR \\
\hline 69 & C-401 & 18.0 & 3 & MR & 16.8 & 3 & MR & 17 & MR \\
\hline 70 & C-404 & 14.6 & 3 & MR & 23.1 & 5 & MS & 19 & MR \\
\hline 71 & C-407 & 28.3 & 5 & MS & 31.4 & 7 & $S$ & 30 & MS \\
\hline 72 & C-417 & 13.6 & 3 & MR & 18.6 & 3 & MR & 16 & MR \\
\hline 73 & C-437 & 20.8 & 5 & MS & 14.3 & 3 & MR & 18 & MR \\
\hline 74 & C-441 & 23.5 & 5 & MS & 18.6 & 3 & MR & 21 & MS \\
\hline 75 & C-448 & 19.6 & 3 & MR & 23.4 & 5 & MS & 22 & MS \\
\hline 76 & C-455 & 18.3 & 3 & MR & 20.8 & 5 & MS & 20 & MR \\
\hline 77 & C-464 & 28.0 & 5 & MS & 21.4 & 5 & MS & 25 & MS \\
\hline 78 & C-470 & 16.9 & 3 & MR & 15 & 3 & MR & 16 & MR \\
\hline 79 & C-473 & 22.5 & 5 & MS & 29.4 & 5 & MS & 26 & MS \\
\hline 80 & C-474 & 28.4 & 5 & MS & 20.6 & 5 & MS & 25 & MS \\
\hline 81 & C-475 & 26.5 & 5 & MS & 23.4 & 5 & MS & 25 & MS \\
\hline 82 & C-479 & 20.8 & 5 & MS & 21.5 & 5 & MS & 21 & MS \\
\hline 83 & C-481 & 16.8 & 3 & MR & 18.6 & 3 & MR & 18 & MR \\
\hline
\end{tabular}




\begin{tabular}{|c|c|c|c|c|c|c|c|c|c|}
\hline S. No & $\begin{array}{c}\text { Entry } \\
\text { No. }\end{array}$ & $\begin{array}{c}\text { Kharif, } \\
2016\end{array}$ & Scale & Status & $\begin{array}{c}\text { Kharif, } \\
2017\end{array}$ & Scale & Status & Mean & Status \\
\hline 84 & C-490 & 22.1 & 5 & MS & 29.4 & 5 & MS & 26 & MS \\
\hline 85 & C-492 & 15.4 & 3 & MR & 16.3 & 3 & MR & 16 & MR \\
\hline 86 & C-497 & 11.6 & 3 & MR & 14.3 & 3 & MR & 13 & MR \\
\hline 87 & C-498 & 28.3 & 5 & MS & 21.5 & 5 & MS & 25 & MS \\
\hline 88 & C-499 & 16.4 & 3 & MR & 20.8 & 5 & MS & 19 & MR \\
\hline 89 & C-502 & 13.9 & 3 & MR & 22.5 & 5 & MS & 18 & MR \\
\hline 90 & C-504 & 14.2 & 3 & MR & 15.8 & 3 & MR & 15 & MR \\
\hline 91 & C-514 & 39.8 & 7 & $\mathrm{~S}$ & 29.8 & 5 & MS & 35 & $\mathrm{~S}$ \\
\hline 92 & C-515 & 22.6 & 5 & MS & 21.8 & 5 & MS & 22 & MS \\
\hline 93 & C-517 & 26.8 & 5 & MS & 24.3 & 5 & MS & 26 & MS \\
\hline 94 & C-518 & 17.6 & 3 & MR & 18.3 & 3 & MR & 18 & MR \\
\hline 95 & C-519 & 18.9 & 3 & MR & 21.8 & 5 & MS & 20 & MR \\
\hline 96 & C-536 & 23.6 & 5 & MS & 29.4 & 5 & MS & 27 & MS \\
\hline 97 & C-537 & 28.4 & 5 & MS & 22.5 & 5 & MS & 25 & MS \\
\hline 98 & C-538 & 31.6 & 7 & $\mathrm{~S}$ & 38.4 & 7 & $\mathrm{~S}$ & 35 & $\mathrm{~S}$ \\
\hline 99 & C-540 & 18.5 & 3 & MR & 19.3 & 3 & MR & 19 & MR \\
\hline 100 & C-550 & 24.6 & 5 & MS & 28.6 & 5 & MS & 27 & MS \\
\hline 101 & C-551 & 20.6 & 5 & MS & 26.5 & 5 & MS & 24 & MS \\
\hline 102 & C-554 & 11.8 & 3 & MR & 16.3 & 3 & MR & 14 & MR \\
\hline 103 & C-556 & 10.8 & 3 & MR & 13.4 & 3 & MR & 12 & MR \\
\hline 104 & C-557 & 21.6 & 5 & MS & 28.6 & 5 & MS & 25 & MS \\
\hline 105 & C-559 & 21.6 & 5 & MS & 29.1 & 5 & MS & 25 & MS \\
\hline 106 & C-560 & 16.4 & 3 & MR & 14.2 & 3 & MR & 15 & MR \\
\hline 107 & C-561 & 38.1 & 7 & $\mathrm{~S}$ & 29 & 5 & MS & 34 & $\mathrm{~S}$ \\
\hline 108 & C-566 & 26.1 & 5 & MS & 20.5 & 5 & MS & 23 & MS \\
\hline 109 & C-575 & 28.3 & 5 & MS & 23.1 & 5 & MS & 26 & MS \\
\hline 110 & C-593 & 19.6 & 3 & MR & 16.8 & 3 & MR & 18 & MR \\
\hline 111 & C-597 & 12.5 & 3 & MR & 16.1 & 3 & MR & 14 & MR \\
\hline 112 & C-599 & 16.2 & 3 & MR & 10.3 & 1 & $\mathrm{R}$ & 13 & MR \\
\hline 113 & C-600 & 28.3 & 5 & MS & 29.1 & 5 & MS & 29 & MS \\
\hline 114 & C-602 & 31.8 & 7 & $\mathrm{~S}$ & 30.4 & 5 & MS & 31 & $S$ \\
\hline 115 & C-603 & 16.9 & 3 & MR & 18.5 & 3 & MR & 18 & MR \\
\hline 116 & C-608 & 28.3 & 5 & MS & 20.4 & 3 & MR & 24 & MS \\
\hline 117 & C-610 & 31.5 & 7 & $S$ & 32.8 & 7 & $S$ & 32 & $S$ \\
\hline 118 & C-621 & 16.4 & 3 & MR & 18.2 & 3 & MR & 17 & MR \\
\hline 119 & C-637 & 18.6 & 3 & MR & 19.0 & 3 & MR & 19 & MR \\
\hline 120 & C-649 & 29.6 & 5 & MS & 30.1 & 5 & MS & 30 & MS \\
\hline 121 & C-651 & 31.8 & 7 & $\mathrm{~S}$ & 32.0 & 7 & $\mathrm{~S}$ & 32 & $\mathrm{~S}$ \\
\hline 122 & C-682 & 19.5 & 3 & MR & 16.4 & 3 & MR & 18 & MR \\
\hline 123 & C-684 & 28.3 & 5 & MS & 23.5 & 5 & MS & 26 & MS \\
\hline 124 & C-685 & 16.9 & 3 & MR & 11.4 & 3 & MR & 14 & MR \\
\hline 125 & C-689 & 18.4 & 3 & MR & 13.5 & 3 & MR & 16 & MR \\
\hline 126 & C-692 & 20.5 & 5 & MS & 16.4 & 3 & MR & 18 & MR \\
\hline
\end{tabular}




\begin{tabular}{|c|c|c|c|c|c|c|c|c|c|}
\hline S. No & $\begin{array}{c}\text { Entry } \\
\text { No. }\end{array}$ & $\begin{array}{c}\text { Kharif, } \\
2016\end{array}$ & Scale & Status & $\begin{array}{c}\text { Kharif, } \\
2017\end{array}$ & Scale & Status & Mean & Status \\
\hline 127 & C-693 & 28.1 & 5 & MS & 26.1 & 5 & MS & 27 & MS \\
\hline 128 & C-697 & 16.3 & 3 & MR & 18.4 & 3 & MR & 17 & MR \\
\hline 129 & C-702 & 26.1 & 5 & MS & 24.0 & 5 & MS & 25 & MS \\
\hline 130 & C-706 & 28.8 & 5 & MS & 20.5 & 5 & MS & 25 & MS \\
\hline 131 & C-713 & 31.4 & 7 & $S$ & 26.3 & 5 & MS & 29 & MS \\
\hline 132 & C-714 & 31.6 & 7 & S & 38.2 & 7 & $\mathrm{~S}$ & 35 & S \\
\hline 133 & C-715 & 30.8 & 7 & $S$ & 26.4 & 5 & MS & 29 & MS \\
\hline 134 & C-727 & 21.8 & 5 & MS & 20.6 & 5 & MS & 21 & MS \\
\hline 135 & C-729 & 28.5 & 5 & MS & 31.0 & 7 & $\mathrm{~S}$ & 30 & MS \\
\hline 136 & C-750 & 21.8 & 5 & MS & 22.6 & 5 & MS & 22 & MS \\
\hline 137 & C-750 & 23.8 & 5 & MS & 20.9 & 5 & MS & 22 & MS \\
\hline 138 & C-753 & 14.5 & 3 & MR & 16.3 & 3 & MR & 15 & MR \\
\hline 139 & C-754 & 28.6 & 5 & MS & 28.9 & 5 & MS & 29 & MS \\
\hline 140 & C-763 & 16.5 & 3 & MR & 10.6 & 3 & MR & 14 & MR \\
\hline 141 & C-766 & 38.4 & 7 & S & 19.8 & 3 & MR & 29 & MS \\
\hline 142 & C-767 & 21.8 & 5 & MS & 22.6 & 5 & MS & 22 & MS \\
\hline 143 & C-775 & 28.3 & 5 & MS & 21.6 & 5 & MS & 25 & MS \\
\hline 144 & C-780 & 25.5 & 5 & MS & 26.3 & 5 & MS & 26 & MS \\
\hline 145 & C-781 & 26.8 & 5 & MS & 19.8 & 3 & MR & 23 & MS \\
\hline 146 & C-782 & 22.1 & 5 & MS & 20.6 & 5 & MS & 21 & MS \\
\hline 147 & C-786 & 19.6 & 3 & MR & 23.5 & 5 & MS & 22 & MS \\
\hline 148 & C-787 & 31.8 & 7 & $\mathrm{~S}$ & 30.5 & 7 & $\mathrm{~S}$ & 31 & $\mathrm{~S}$ \\
\hline 149 & C-788 & 26.8 & 5 & MS & 28.3 & 5 & MS & 28 & MS \\
\hline 150 & C-790 & 33.9 & 7 & $\mathrm{~S}$ & 30.5 & 7 & $\mathrm{~S}$ & 32 & $\mathrm{~S}$ \\
\hline 151 & C-792 & 31.8 & 7 & $S$ & 30.6 & 7 & $S$ & 31 & $S$ \\
\hline 152 & C-793 & 29.6 & 5 & MS & 21.8 & 5 & MS & 26 & MS \\
\hline 153 & C-794 & 31.2 & 7 & $S$ & 29.6 & 5 & MS & 30 & MS \\
\hline 154 & C-795 & 22.8 & 5 & MS & 24.3 & 5 & MS & 24 & MS \\
\hline 155 & C-797 & 19.3 & 3 & MR & 22.8 & 5 & MS & 21 & MS \\
\hline 156 & C-798 & 20.8 & 5 & MS & 18.6 & 3 & MR & 20 & MR \\
\hline 157 & C-804 & 31.5 & 7 & $S$ & 16.5 & 3 & MR & 24 & MS \\
\hline 158 & C-808 & 24.3 & 5 & MS & 20.8 & 5 & MS & 23 & MS \\
\hline 159 & C-810 & 28.5 & 5 & MS & 21.5 & 5 & MS & 25 & MS \\
\hline 160 & C-812 & 34.8 & 7 & $S$ & 26.8 & 5 & MS & 31 & $\mathrm{~S}$ \\
\hline 161 & C-828 & 18.6 & 3 & MR & 16.9 & 3 & MR & 18 & MR \\
\hline 162 & C-844 & 31.6 & 7 & $\mathrm{~S}$ & 30.5 & 7 & $\mathrm{~S}$ & 31 & $\mathrm{~S}$ \\
\hline 163 & C-851 & 22.9 & 5 & MS & 26.5 & 5 & MS & 25 & MS \\
\hline 164 & C-858 & 10.5 & 3 & MR & 10.8 & 3 & MR & 11 & MR \\
\hline 165 & C-864 & 18.3 & 3 & MR & 26.4 & 5 & MS & 22 & MS \\
\hline 166 & C-870 & 26.8 & 5 & MS & 30.4 & 5 & MS & 29 & MS \\
\hline 167 & C-878 & 34.9 & 7 & $\mathrm{~S}$ & 32.6 & 7 & $S$ & 34 & $\mathrm{~S}$ \\
\hline 168 & C-879 & 18.4 & 3 & MR & 18.2 & 3 & MR & 18 & MR \\
\hline 169 & C-884 & 14.9 & 3 & MR & 16.3 & 3 & MR & 16 & MR \\
\hline
\end{tabular}




\begin{tabular}{|c|c|c|c|c|c|c|c|c|c|}
\hline S. No & $\begin{array}{c}\text { Entry } \\
\text { No. }\end{array}$ & $\begin{array}{c}\text { Kharif, } \\
2016\end{array}$ & Scale & Status & $\begin{array}{c}\text { Kharif } \\
2017\end{array}$ & Scale & Status & Mean & Status \\
\hline 170 & C-891 & 28.5 & 5 & MS & 29.0 & 5 & MS & 29 & MS \\
\hline 171 & C-901 & 16.9 & 3 & MR & 13.4 & 3 & MR & 15 & MR \\
\hline 172 & C-902 & 23.4 & 5 & MS & 19.3 & 3 & MR & 21 & MS \\
\hline 173 & C-903 & 16.8 & 3 & MR & 13.4 & 3 & MR & 15 & MR \\
\hline 174 & C-1165 & 21.8 & 5 & MS & 26.8 & 5 & MS & 24 & MS \\
\hline 175 & C-1167 & 18.6 & 3 & MR & 19.3 & 3 & MR & 19 & MR \\
\hline 176 & C-1170 & 11.4 & 3 & MR & 20.6 & 5 & MS & 16 & MR \\
\hline 177 & C-1172 & 18.3 & 3 & MR & 11.5 & 3 & MR & 15 & MR \\
\hline 178 & C-1175 & 29.3 & 5 & MS & 26.9 & 5 & MS & 28 & MS \\
\hline 179 & C-1176 & 16.4 & 3 & MR & 20.6 & 5 & MS & 19 & MR \\
\hline 180 & C-1179 & 19.8 & 3 & MR & 22.0 & 5 & MS & 21 & MS \\
\hline 181 & C-1180 & 30.1 & 5 & MS & 26.3 & 5 & MS & 28 & MS \\
\hline 182 & C-1181 & 38.3 & 7 & $\mathrm{~S}$ & 21.8 & 5 & MS & 30 & MS \\
\hline 183 & C-1185 & 29.1 & 5 & MS & 30.5 & 7 & $\mathrm{~S}$ & 30 & MS \\
\hline 184 & C-1199 & 19.8 & 3 & MR & 20.1 & 3 & MR & 20 & MR \\
\hline 185 & C-1205 & 23.5 & 5 & MS & 26.3 & 5 & MS & 25 & MS \\
\hline 186 & C-1226 & 26.5 & 5 & MS & 16.8 & 3 & MR & 22 & MS \\
\hline 187 & C-1237 & 19.8 & 3 & MR & 16.4 & 3 & MR & 18 & MR \\
\hline 188 & C-1241 & 20.8 & 5 & MS & 16.9 & 3 & MR & 19 & MR \\
\hline 189 & C-1247 & 16.3 & 3 & MR & 13.2 & 3 & MR & 15 & MR \\
\hline 190 & C-1248 & 21.5 & 5 & MS & 20.8 & 5 & MS & 21 & MS \\
\hline 191 & C-1249 & 22.9 & 5 & MS & 18.3 & 3 & MR & 21 & MS \\
\hline 192 & C- 1257 & 28.3 & 5 & MS & 16.3 & 3 & MR & 22 & MS \\
\hline 193 & C-1259 & 19.6 & 3 & MR & 20.4 & 3 & MR & 20 & MR \\
\hline 194 & C-1289 & 21.3 & 5 & MS & 21.6 & 5 & MS & 21 & MS \\
\hline 195 & C-1320 & 23.5 & 5 & MS & 20.4 & 3 & MR & 22 & MS \\
\hline 196 & C-1328 & 26.3 & 5 & MS & 26.3 & 5 & MS & 26 & MS \\
\hline 197 & C-1372 & 11.9 & 3 & MR & 10.8 & 3 & MR & 11 & MR \\
\hline 198 & C-1378 & 21.8 & 5 & MS & 19.9 & 3 & MR & 21 & MS \\
\hline 199 & C-1391 & 30.5 & 7 & $\mathrm{~S}$ & 41.5 & 7 & $S$ & 36 & $S$ \\
\hline 200 & C-1394 & 16.8 & 3 & MR & 21.8 & 5 & MS & 19 & MR \\
\hline 201 & C-1397 & 28.5 & 5 & MS & 16.9 & 3 & MR & 23 & MS \\
\hline 202 & C-1398 & 31.5 & 7 & $\mathrm{~S}$ & 29.8 & 5 & MS & 31 & $S$ \\
\hline 203 & C-1406 & 21.4 & 5 & MS & 25.8 & 5 & MS & 24 & MS \\
\hline 204 & C-1430 & 20.6 & 5 & MS & 30.0 & 5 & MS & 25 & MS \\
\hline 205 & C-1433 & 18.3 & 3 & MR & 16.9 & 3 & MR & 18 & MR \\
\hline 206 & C-1436 & 31.8 & 7 & $\mathrm{~S}$ & 33.4 & 7 & S & 33 & S \\
\hline 207 & C-1439 & 29.6 & 5 & MS & 30.8 & 7 & S & 30 & MS \\
\hline 208 & C-1448 & 23.4 & 5 & MS & 24.6 & 5 & MS & 24 & MS \\
\hline 209 & C-1449 & 19.8 & 3 & MR & 20.5 & 5 & MS & 20 & MR \\
\hline 210 & C-1453 & 32.5 & 7 & S & 29.9 & 5 & MS & 31 & S \\
\hline 211 & C-1463 & 28.3 & 5 & MS & 21.9 & 5 & MS & 25 & MS \\
\hline 212 & C-1464 & 16.9 & 3 & MR & 16.4 & 3 & MR & 17 & MR \\
\hline 213 & C-1474 & 29.8 & 5 & MS & 20.3 & 3 & MR & 25 & MS \\
\hline 214 & C-1475 & 31.8 & 7 & S & 29.6 & 5 & MS & 31 & S \\
\hline 215 & C- 1548 & 21.6 & 5 & MS & 20.0 & 3 & MR & 21 & MS \\
\hline \multirow[t]{2}{*}{ Checks } & TN1 & 42.2 & 7 & S & 35.1 & 7 & $\mathrm{~S}$ & 39 & S \\
\hline & BPT 5204 & 32.8 & 7 & S & 26.9 & 5 & MS & 30 & MS \\
\hline \multicolumn{2}{|c|}{ Mean } & 22.94 & - & - & 22.39 & - & - & 22.67 & - \\
\hline \multicolumn{2}{|c|}{ Std. Dev. } & 8.28 & - & - & 9.61 & - & - & 8.95 & - \\
\hline \multicolumn{2}{|c|}{ Std. Error } & 0.56 & - & - & 0.65 & - & - & 0.61 & - \\
\hline \multicolumn{2}{|c|}{ CV (\%) } & 36.07 & - & - & 42.88 & - & - & 57.51 & - \\
\hline
\end{tabular}


Table.4 Field identification of rice genotypes against yellow stem borer, in terms of per cent white ears

\begin{tabular}{|c|c|c|c|c|c|c|c|c|c|}
\hline S.No. & $\begin{array}{c}\text { Entry } \\
\text { No. }\end{array}$ & $\begin{array}{c}\text { Kharif, } \\
2016\end{array}$ & Scale & Status & $\begin{array}{c}\text { Kharif, } \\
2017\end{array}$ & Scale & Status & Mean & Status \\
\hline 1 & C- -08 & 11.3 & 5 & MS & 10.3 & 3 & MR & 11 & MS \\
\hline 2 & C-17 & 14.3 & 5 & MS & 13.5 & 5 & MS & 14 & MS \\
\hline 3 & C-27 & 8.6 & 3 & MR & 7.5 & 3 & MR & 8 & MR \\
\hline 4 & C-30 & 10.3 & 3 & MR & 8.9 & 3 & MR & 10 & MR \\
\hline 5 & C-37 & 6.8 & 3 & MR & 6.0 & 3 & MR & 6 & MR \\
\hline 6 & C-53 & 11.9 & 5 & MS & 12.0 & 5 & MS & 12 & MS \\
\hline 7 & C-55 & 15.2 & 5 & MS & 13.4 & 5 & MS & 14 & MS \\
\hline 8 & C-58 & 12.3 & 5 & MS & 10.3 & 3 & MR & 11 & MS \\
\hline 9 & C-64 & 14.0 & 5 & MS & 18.2 & 7 & $S$ & 16 & $S$ \\
\hline 10 & C-86 & 11.3 & 5 & MS & 6.8 & 3 & MR & 9 & MR \\
\hline 11 & C-115 & 8.6 & 3 & MR & 10.2 & 3 & MR & 9 & MR \\
\hline 12 & C-124 & 9.3 & 3 & MR & 8.6 & 3 & MR & 9 & MR \\
\hline 13 & C-133 & 10.4 & 3 & MR & 10.1 & 3 & MR & 10 & MR \\
\hline 14 & C-140 & 16.1 & 7 & $\mathrm{~S}$ & 17.2 & 7 & $S$ & 17 & S \\
\hline 15 & C-141 & 10.9 & 5 & MS & 16.1 & 7 & $S$ & 14 & MS \\
\hline 16 & C-144 & 13.4 & 5 & MS & 11.5 & 5 & MS & 12 & MS \\
\hline 17 & C-152 & 18.0 & 7 & $S$ & 12.5 & 5 & MS & 15 & MS \\
\hline 18 & C-170 & 16.4 & 7 & $S$ & 18.5 & 7 & $\mathrm{~S}$ & 17 & $S$ \\
\hline 19 & C-171 & 8.9 & 3 & MR & 6.5 & 3 & MR & 8 & MR \\
\hline 20 & C-178 & 7.3 & 3 & MR & 7.0 & 3 & MR & 7 & MR \\
\hline 21 & C-202 & 15.4 & 5 & MS & 11.0 & 5 & MS & 13 & MS \\
\hline 22 & C-207 & 13.9 & 5 & MS & 19.5 & 7 & S & 17 & S \\
\hline 23 & C-221 & 10.8 & 5 & MS & 11.3 & 5 & MS & 11 & MS \\
\hline 24 & C-228 & 13.9 & 5 & MS & 14.0 & 5 & MS & 14 & MS \\
\hline 25 & C-237 & 16.4 & 7 & $S$ & 13.8 & 5 & MS & 15 & MS \\
\hline 26 & C-240 & 13.2 & 5 & MS & 12.8 & 5 & MS & 13 & MS \\
\hline 27 & $\mathrm{C}-250$ & 10.5 & 5 & MS & 11.5 & 5 & MS & 11 & MS \\
\hline 28 & C-269 & 12.6 & 5 & MS & 16.8 & 7 & $S$ & 15 & MS \\
\hline 29 & $\mathrm{C}-270$ & 25.0 & 7 & $\mathrm{~S}$ & 10.3 & 3 & MR & 18 & $S$ \\
\hline 30 & $\mathrm{C}-273$ & 10.4 & 3 & MR & 11.0 & 5 & MS & 11 & MS \\
\hline 31 & C-275 & 10.4 & 3 & MR & 10.5 & 5 & MS & 10 & MR \\
\hline 32 & C-280 & 9.8 & 3 & MR & 10.4 & 3 & MR & 10 & MR \\
\hline 33 & C-288 & 6.9 & 3 & MR & 6.9 & 3 & MR & 7 & MR \\
\hline 34 & C-306 & 8.0 & 3 & MR & 5.3 & 1 & $\mathrm{R}$ & 7 & MR \\
\hline 35 & C-319 & 10.3 & 3 & MR & 7.1 & 3 & MR & 9 & MR \\
\hline 36 & C-321 & 14.2 & 5 & MS & 11.5 & 5 & MS & 13 & MS \\
\hline 37 & C-324 & 8.5 & 3 & MR & 6.8 & 3 & MR & 8 & MR \\
\hline
\end{tabular}




\begin{tabular}{|c|c|c|c|c|c|c|c|c|c|}
\hline S.No. & $\begin{array}{c}\text { Entry } \\
\text { No. }\end{array}$ & $\begin{array}{c}\text { Kharif, } \\
2016\end{array}$ & Scale & Status & $\begin{array}{c}\text { Kharif, } \\
2017\end{array}$ & Scale & Status & Mean & Status \\
\hline 38 & C-328 & 11.8 & 5 & MS & 12 & 5 & MS & 12 & MS \\
\hline 39 & C-330 & 16.3 & 7 & $\mathrm{~S}$ & 14.3 & 5 & MS & 15 & MS \\
\hline 40 & C-342 & 12.8 & 5 & MS & 10.9 & 5 & MS & 12 & MS \\
\hline 41 & C-343 & 16.4 & 7 & $S$ & 10.8 & 5 & MS & 14 & MS \\
\hline 42 & C-346 & 10.4 & 3 & MR & 11.6 & 5 & MS & 11 & MS \\
\hline 43 & C-349 & 13.6 & 5 & MS & 14.8 & 5 & MS & 14 & MS \\
\hline 44 & C-350 & 10.3 & 3 & MR & 16.3 & 7 & $S$ & 13 & MS \\
\hline 45 & C-352 & 6.9 & 3 & MR & 5.6 & 3 & MR & 6 & MR \\
\hline 46 & C-354 & 15.1 & 5 & MS & 14.3 & 5 & MS & 15 & MS \\
\hline 47 & C-358 & 18.3 & 7 & S & 12.6 & 5 & MS & 15 & MS \\
\hline 48 & C-361 & 10.3 & 3 & MR & 11.9 & 5 & MS & 11 & MS \\
\hline 49 & C-362 & 18.6 & 7 & $S$ & 19.3 & 7 & S & 19 & $S$ \\
\hline 50 & C-364 & 14.3 & 5 & MS & 16.2 & 7 & $S$ & 15 & MS \\
\hline 51 & C-365 & 10.9 & 5 & MS & 9.8 & 3 & MR & 10 & MR \\
\hline 52 & C-368 & 21.6 & 7 & $\mathrm{~S}$ & 16.3 & 7 & S & 19 & $S$ \\
\hline 53 & C-369 & 6.4 & 3 & MR & 5.3 & 1 & $\mathrm{R}$ & 6 & MR \\
\hline 54 & C-372 & 10.2 & 3 & MR & 3.9 & 1 & $\mathrm{R}$ & 7 & MR \\
\hline 55 & C-373 & 7.3 & 3 & MR & 6.4 & 3 & MR & 7 & MR \\
\hline 56 & C-374 & 6.0 & 3 & MR & 5.1 & 1 & $\mathrm{R}$ & 6 & MR \\
\hline 57 & C-377 & 8.1 & 3 & MR & 6.1 & 3 & MR & 7 & MR \\
\hline 58 & C-378 & 8.4 & 3 & MR & 9.3 & 3 & MR & 9 & MR \\
\hline 59 & C-380 & 6.5 & 3 & MR & 6.0 & 3 & MR & 6 & MR \\
\hline 60 & C-384 & 7.9 & 3 & MR & 9.9 & 3 & MR & 9 & MR \\
\hline 61 & C-386 & 10.9 & 5 & MS & 9.3 & 3 & MR & 10 & MR \\
\hline 62 & C-388 & 15.3 & 5 & MS & 11.0 & 5 & MS & 13 & MS \\
\hline 63 & C-390 & 16.8 & 7 & S & 17.3 & 7 & S & 17 & $S$ \\
\hline 64 & C-391 & 10.9 & 5 & MS & 16.2 & 7 & $S$ & 14 & MS \\
\hline 65 & C-393 & 16.3 & 7 & S & 14.3 & 5 & MS & 15 & MS \\
\hline 66 & C-394 & 18.2 & 7 & $S$ & 16.3 & 7 & $S$ & 17 & $S$ \\
\hline 67 & C-396 & 10.2 & 3 & MR & 10.8 & 5 & MS & 11 & MS \\
\hline 68 & C-400 & 9.4 & 3 & MR & 8.6 & 3 & MR & 9 & MR \\
\hline 69 & C-401 & 9.8 & 3 & MR & 9.0 & 3 & MR & 9 & MR \\
\hline 70 & C-404 & 15.1 & 5 & MS & 14.2 & 5 & MS & 15 & MS \\
\hline 71 & C-407 & 18.3 & 7 & S & 10.3 & 3 & MR & 14 & MS \\
\hline 72 & C-417 & 20.3 & 7 & $S$ & 16.9 & 7 & $S$ & 19 & $S$ \\
\hline 73 & C-437 & 10.8 & 5 & MS & 9.8 & 3 & MR & 10 & MR \\
\hline 74 & C-441 & 10.3 & 3 & MR & 6.4 & 3 & MR & 8 & MR \\
\hline 75 & C-448 & 12.4 & 5 & MS & 8.4 & 3 & MR & 10 & MR \\
\hline 76 & C-455 & 11.4 & 5 & MS & 9.9 & 3 & MR & 11 & MS \\
\hline 77 & C-464 & 19.2 & 7 & S & 14.3 & 5 & MS & 17 & $S$ \\
\hline 78 & C-470 & 6.4 & 3 & MR & 6 & 3 & MR & 6 & MR \\
\hline 79 & C-473 & 8.3 & 3 & MR & 9.2 & 3 & MR & 9 & MR \\
\hline
\end{tabular}




\begin{tabular}{|c|c|c|c|c|c|c|c|c|c|}
\hline S.No. & $\begin{array}{c}\text { Entry } \\
\text { No. }\end{array}$ & $\begin{array}{c}\text { Kharif, } \\
2016\end{array}$ & Scale & Status & $\begin{array}{c}\text { Kharif, } \\
2017\end{array}$ & Scale & Status & Mean & Status \\
\hline 80 & C-474 & 10.9 & 5 & MS & 11.4 & 5 & MS & 11 & MS \\
\hline 81 & C-475 & 14.2 & 5 & MS & 12.1 & 5 & MS & 13 & MS \\
\hline 82 & C-479 & 16.3 & 7 & $\mathrm{~S}$ & 14.3 & 5 & MS & 15 & MS \\
\hline 83 & C-481 & 10.4 & 3 & MR & 9.8 & 3 & MR & 10 & MR \\
\hline 84 & C-490 & 31.5 & 9 & HS & 29.3 & 9 & HS & 30 & HS \\
\hline 85 & C-492 & 7.3 & 3 & MR & 4.2 & 1 & $\mathrm{R}$ & 6 & MR \\
\hline 86 & C-497 & 10.8 & 5 & MS & 10.1 & 3 & MR & 10 & MR \\
\hline 87 & C-498 & 11.4 & 5 & MS & 12.8 & 5 & MS & 12 & MS \\
\hline 88 & C-499 & 8.0 & 3 & MR & 6.3 & 3 & MR & 7 & MR \\
\hline 89 & C-502 & 7.5 & 3 & MR & 6.0 & 3 & MR & 7 & MR \\
\hline 90 & C-504 & 10.4 & 3 & MR & 10.0 & 3 & MR & 10 & MR \\
\hline 91 & C-514 & 11.8 & 5 & MS & 12.0 & 5 & MS & 12 & MS \\
\hline 92 & C-515 & 10.3 & 3 & MR & 9.6 & 3 & MR & 10 & MR \\
\hline 93 & C-517 & 12.4 & 5 & MS & 6.8 & 3 & MR & 10 & MR \\
\hline 94 & C-518 & 7.8 & 3 & MR & 8.3 & 3 & MR & 8 & MR \\
\hline 95 & C-519 & 8.0 & 3 & MR & 8.5 & 3 & MR & 8 & MR \\
\hline 96 & C-536 & 10.4 & 3 & MR & 11.5 & 5 & MS & 11 & MS \\
\hline 97 & C-537 & 6.9 & 3 & MR & 6.8 & 3 & MR & 7 & MR \\
\hline 98 & C-538 & 12.2 & 5 & MS & 10.4 & 3 & MR & 11 & MS \\
\hline 99 & C-540 & 9.3 & 3 & MR & 13.5 & 5 & MS & 11 & MS \\
\hline 100 & C-550 & 10.8 & 5 & MS & 11.5 & 5 & MS & 11 & MS \\
\hline 101 & C-551 & 11.9 & 5 & MS & 10.9 & 5 & MS & 11 & MS \\
\hline 102 & C-554 & 10.5 & 5 & MS & 11.5 & 5 & MS & 11 & MS \\
\hline 103 & C-556 & 6.3 & 3 & MR & 4.8 & 1 & $\mathrm{R}$ & 6 & MR \\
\hline 104 & C-557 & 13.8 & 5 & MS & 10.3 & 3 & MR & 12 & MS \\
\hline 105 & C-559 & 15.3 & 5 & MS & 11.4 & 5 & MS & 13 & MS \\
\hline 106 & C-560 & 6.3 & 3 & MR & 8.4 & 3 & MR & 7 & MR \\
\hline 107 & C-561 & 18.0 & 7 & $S$ & 16.8 & 7 & $S$ & 17 & $S$ \\
\hline 108 & C-566 & 11.5 & 5 & MS & 12.8 & 5 & MS & 12 & MS \\
\hline 109 & C-575 & 14.3 & 5 & MS & 9.6 & 3 & MR & 12 & MS \\
\hline 110 & C-593 & 4.2 & 1 & $\mathrm{R}$ & 9.0 & 3 & MR & 7 & MR \\
\hline 111 & C-597 & 10.3 & 3 & MR & 9.3 & 3 & MR & 10 & MR \\
\hline 112 & C-599 & 15.3 & 5 & MS & 11.8 & 5 & MS & 14 & MS \\
\hline 113 & C-600 & 12.6 & 5 & MS & 10.3 & 3 & MR & 11 & MS \\
\hline 114 & C-602 & 10.6 & 5 & MS & 9.8 & 3 & MR & 10 & MR \\
\hline 115 & C-603 & 7.5 & 3 & MR & 8.4 & 3 & MR & 8 & MR \\
\hline 116 & C-608 & 8.9 & 3 & MR & 9.3 & 3 & MR & 9 & MR \\
\hline 117 & C-610 & 10.3 & 3 & MR & 9.6 & 3 & MR & 10 & MR \\
\hline 118 & C-621 & 9.2 & 3 & MR & 8.9 & 3 & MR & 9 & MR \\
\hline 119 & C-637 & 8.6 & 3 & MR & 7.3 & 3 & MR & 8 & MR \\
\hline 120 & C-649 & 6.9 & 3 & MR & 4.9 & 1 & $\mathrm{R}$ & 6 & MR \\
\hline 121 & C-651 & 17.9 & 7 & $S$ & 16.3 & 7 & $S$ & 17 & $S$ \\
\hline
\end{tabular}




\begin{tabular}{|c|c|c|c|c|c|c|c|c|c|}
\hline S.No. & $\begin{array}{c}\text { Entry } \\
\text { No. }\end{array}$ & $\begin{array}{c}\text { Kharif, } \\
2016\end{array}$ & Scale & Status & $\begin{array}{c}\text { Kharif, } \\
2017\end{array}$ & Scale & Status & Mean & Status \\
\hline 122 & C-682 & 10.4 & 3 & MR & 5.6 & 3 & MR & 8 & MR \\
\hline 123 & C-684 & 13.8 & 5 & MS & 11.4 & 5 & MS & 13 & MS \\
\hline 124 & C-685 & 6.5 & 3 & MR & 3.5 & 1 & $\mathrm{R}$ & 5 & $\mathrm{R}$ \\
\hline 125 & C-689 & 10.3 & 3 & MR & 6.0 & 3 & MR & 8 & MR \\
\hline 126 & C-692 & 15.8 & 7 & $S$ & 8.1 & 3 & MR & 12 & MS \\
\hline 127 & C-693 & 10.3 & 3 & MR & 9.3 & 3 & MR & 10 & MR \\
\hline 128 & C-697 & 6.4 & 3 & MR & 5.0 & 1 & $\mathrm{R}$ & 6 & MR \\
\hline 129 & C-702 & 5.0 & 1 & $\mathrm{R}$ & 6.3 & 3 & MR & 6 & MR \\
\hline 130 & C-706 & 16.4 & 7 & $S$ & 10.4 & 3 & MR & 13 & MS \\
\hline 131 & C-713 & 19.3 & 7 & S & 11.6 & 5 & MS & 15 & MS \\
\hline 132 & C-714 & 18.6 & 7 & $S$ & 14.2 & 5 & MS & 16 & $S$ \\
\hline 133 & C-715 & 10.9 & 5 & MS & 9.9 & 3 & MR & 10 & MR \\
\hline 134 & C-727 & 18.3 & 7 & $\mathrm{~S}$ & 21.8 & 7 & $\mathrm{~S}$ & 20 & $\mathrm{~S}$ \\
\hline 135 & C-729 & 10.4 & 3 & MR & 9.5 & 3 & MR & 10 & MR \\
\hline 136 & C-750 & 10.4 & 3 & MR & 9.3 & 3 & MR & 10 & MR \\
\hline 137 & C-750 & 13.4 & 5 & MS & 14.0 & 5 & MS & 14 & MS \\
\hline 138 & C-753 & 6.3 & 3 & MR & 6.9 & 3 & MR & 7 & MR \\
\hline 139 & C-754 & 10.8 & 5 & MS & 11.3 & 5 & MS & 11 & MS \\
\hline 140 & C-763 & 7.3 & 3 & MR & 8.0 & 3 & MR & 8 & MR \\
\hline 141 & C-766 & 8.9 & 3 & MR & 7.3 & 3 & MR & 8 & MR \\
\hline 142 & C-767 & 10.6 & 5 & MS & 11.8 & 5 & MS & 11 & MS \\
\hline 143 & C-775 & 10.3 & 3 & MR & 12.5 & 5 & MS & 11 & MS \\
\hline 144 & C-780 & 11.4 & 5 & MS & 10.8 & 5 & MS & 11 & MS \\
\hline 145 & C-781 & 10.1 & 3 & MR & 9.5 & 3 & MR & 10 & MR \\
\hline 146 & C-782 & 8.3 & 3 & MR & 6.9 & 3 & MR & 8 & MR \\
\hline 147 & C-786 & 9.3 & 3 & MR & 8.2 & 3 & MR & 9 & MR \\
\hline 148 & C-787 & 9.4 & 3 & MR & 15.6 & 7 & $\mathrm{~S}$ & 13 & MS \\
\hline 149 & C-788 & 16.5 & 7 & $S$ & 19.2 & 7 & $S$ & 18 & $S$ \\
\hline 150 & C-790 & 19.2 & 7 & $S$ & 10.3 & 3 & MR & 15 & MS \\
\hline 151 & C-792 & 18.3 & 7 & S & 10.8 & 5 & MS & 15 & MS \\
\hline 152 & C-793 & 10.6 & 5 & MS & 8.8 & 3 & MR & 10 & MR \\
\hline 153 & C-794 & 9.8 & 3 & MR & 9.0 & 3 & MR & 9 & MR \\
\hline 154 & C-795 & 11.6 & 5 & MS & 12.4 & 5 & MS & 12 & MS \\
\hline 155 & C-797 & 13.8 & 5 & MS & 10.6 & 5 & MS & 12 & MS \\
\hline 156 & C-798 & 14.1 & 5 & MS & 6.8 & 3 & MR & 10 & MR \\
\hline 157 & C-804 & 10.8 & 5 & MS & 3.9 & 1 & $\mathrm{R}$ & 7 & MR \\
\hline 158 & C-808 & 11.5 & 5 & MS & 8.4 & 3 & MR & 10 & MR \\
\hline 159 & C-810 & 16.8 & 7 & $S$ & 3.9 & 1 & $\mathrm{R}$ & 10 & MR \\
\hline 160 & C-812 & 18.3 & 7 & $\mathrm{~S}$ & 6.4 & 3 & MR & 12 & MS \\
\hline 161 & C-828 & 9.8 & 3 & MR & 5.3 & 1 & $\mathrm{R}$ & 8 & MR \\
\hline 162 & C-844 & 11.9 & 5 & MS & 9.1 & 3 & MR & 11 & MS \\
\hline 163 & C-851 & 13.8 & 5 & MS & 16.2 & 7 & $S$ & 15 & MS \\
\hline
\end{tabular}




\begin{tabular}{|c|c|c|c|c|c|c|c|c|c|}
\hline S.No. & $\begin{array}{c}\text { Entry } \\
\text { No. }\end{array}$ & $\begin{array}{c}\text { Kharif, } \\
2016\end{array}$ & Scale & Status & $\begin{array}{c}\text { Kharif, } \\
2017\end{array}$ & Scale & Status & Mean & Status \\
\hline 164 & C-858 & 4.3 & 1 & $\mathrm{R}$ & 2.9 & 1 & $\mathrm{R}$ & 4 & $\mathrm{R}$ \\
\hline 165 & C-864 & 10.2 & 3 & MR & 3.3 & 1 & $\mathrm{R}$ & 7 & MR \\
\hline 166 & C-870 & 13.9 & 5 & MS & 10 & 3 & MR & 12 & MS \\
\hline 167 & C-878 & 16.3 & 7 & $\mathrm{~S}$ & 9.8 & 3 & MR & 13 & MS \\
\hline 168 & C-879 & 10.9 & 5 & MS & 11.2 & 5 & MS & 11 & MS \\
\hline 169 & C-884 & 8.4 & 3 & MR & 8.0 & 3 & MR & 8 & MR \\
\hline 170 & C-891 & 9.8 & 3 & MR & 11.4 & 5 & MS & 11 & MS \\
\hline 171 & C-901 & 5.6 & 3 & MR & 5.0 & 1 & $\mathrm{R}$ & 5 & $\mathrm{R}$ \\
\hline 172 & C-902 & 6.8 & 3 & MR & 4.8 & 1 & $\mathrm{R}$ & 6 & MR \\
\hline 173 & C-903 & 4.3 & 1 & $\mathrm{R}$ & 4.0 & 1 & $\mathrm{R}$ & 4 & $\mathrm{R}$ \\
\hline 174 & C-1165 & 10.4 & 3 & MR & 6.8 & 3 & MR & 9 & MR \\
\hline 175 & C-1167 & 6.3 & 3 & MR & 6.3 & 3 & MR & 6 & MR \\
\hline 176 & C-1170 & 4.4 & 1 & $\mathrm{R}$ & 10.3 & 3 & MR & 7 & MR \\
\hline 177 & C-1172 & 6 & 3 & MR & 7.5 & 3 & MR & 7 & MR \\
\hline 178 & C-1175 & 10.4 & 3 & MR & 10.3 & 3 & MR & 10 & MR \\
\hline 179 & C-1176 & 4.3 & 1 & $\mathrm{R}$ & 4.3 & 1 & $\mathrm{R}$ & 4 & $\mathrm{R}$ \\
\hline 180 & C-1179 & 4.9 & 1 & $\mathrm{R}$ & 2.8 & 1 & $\mathrm{R}$ & 4 & $\mathrm{R}$ \\
\hline 181 & C-1180 & 10.8 & 5 & MS & 10.3 & 3 & MR & 11 & MS \\
\hline 182 & C-1181 & 13.9 & 5 & MS & 14.2 & 5 & MS & 14 & MS \\
\hline 183 & C-1185 & 10.4 & 3 & MR & 16.8 & 7 & $S$ & 14 & MS \\
\hline 184 & C-1199 & 9.2 & 3 & MR & 10.2 & 3 & MR & 10 & MR \\
\hline 185 & C-1205 & 11.8 & 5 & MS & 10.9 & 5 & MS & 11 & MS \\
\hline 186 & C-1226 & 10.2 & 3 & MR & 6.4 & 3 & MR & 8 & MR \\
\hline 187 & C-1237 & 6.8 & 3 & MR & 2.8 & 1 & $\mathrm{R}$ & 5 & $\mathrm{R}$ \\
\hline 188 & C-1241 & 4.3 & 1 & $\mathrm{R}$ & 4.0 & 1 & $\mathrm{R}$ & 4 & $\mathrm{R}$ \\
\hline 189 & C-1247 & 6.1 & 3 & MR & 4.1 & 1 & $\mathrm{R}$ & 5 & $\mathrm{R}$ \\
\hline 190 & C-1248 & 10.4 & 3 & MR & 3.9 & 1 & $\mathrm{R}$ & 7 & MR \\
\hline 191 & C-1249 & 9.8 & 3 & MR & 6.8 & 3 & MR & 8 & MR \\
\hline 192 & C-1257 & 6.3 & 3 & MR & 9.4 & 3 & MR & 8 & MR \\
\hline 193 & C-1259 & 9 & 3 & MR & 9.3 & 3 & MR & 9 & MR \\
\hline 194 & C-1289 & 9.8 & 3 & MR & 6.9 & 3 & MR & 8 & MR \\
\hline 195 & C-1320 & 10.3 & 3 & MR & 11.2 & 5 & MS & 11 & MS \\
\hline 196 & C-1328 & 11.8 & 5 & MS & 10.0 & 3 & MR & 11 & MS \\
\hline 197 & C-1372 & 4 & 1 & $\mathrm{R}$ & 3.2 & 1 & $\mathrm{R}$ & 4 & $\mathrm{R}$ \\
\hline 198 & C-1378 & 6.9 & 3 & MR & 3.5 & 1 & $\mathrm{R}$ & 5 & $\mathrm{R}$ \\
\hline 199 & C-1391 & 18.2 & 7 & $\mathrm{~S}$ & 4.8 & 1 & $\mathrm{R}$ & 12 & MS \\
\hline 200 & C-1394 & 10.5 & 5 & MS & 6.8 & 3 & MR & 9 & MR \\
\hline 201 & C-1397 & 6.2 & 3 & MR & 6.0 & 3 & MR & 6 & MR \\
\hline 202 & C-1398 & 4.9 & 1 & $\mathrm{R}$ & 2.3 & 1 & $\mathrm{R}$ & 4 & $\mathrm{R}$ \\
\hline 203 & C-1406 & 11.8 & 5 & MS & 6.9 & 3 & MR & 9 & MR \\
\hline 204 & C-1430 & 19.3 & 7 & $\mathrm{~S}$ & 11.5 & 5 & MS & 15 & MS \\
\hline 205 & C-1433 & 2.4 & 1 & $\mathrm{R}$ & 3.5 & 1 & $\mathrm{R}$ & 3 & $\mathrm{R}$ \\
\hline
\end{tabular}




\begin{tabular}{|c|c|c|c|c|c|c|c|c|c|}
\hline $\mathbf{2 0 6}$ & C-1436 & 18.6 & 7 & S & 21.0 & 7 & S & 20 & S \\
\hline $\mathbf{2 0 7}$ & C-1439 & 7.4 & 3 & MR & 8.3 & 3 & MR & 8 & MR \\
\hline $\mathbf{2 0 8}$ & C-1448 & 10.6 & 5 & MS & 10.0 & 3 & MR & 10 & MR \\
\hline $\mathbf{2 0 9}$ & C-1449 & 10.5 & 5 & MS & 6.8 & 3 & MR & 9 & MR \\
\hline $\mathbf{2 1 0}$ & C-1453 & 10.3 & 3 & MR & 8.3 & 3 & MR & 9 & MR \\
\hline $\mathbf{2 1 1}$ & C-1463 & 10.9 & 5 & MS & 6.8 & 3 & MR & 9 & MR \\
\hline $\mathbf{2 1 2}$ & C-1464 & 3.6 & 1 & R & 2.5 & 1 & R & 3 & R \\
\hline $\mathbf{2 1 3}$ & C-1474 & 16.8 & 7 & S & 8.6 & 3 & MR & 13 & MS \\
\hline $\mathbf{2 1 4}$ & C-1475 & 10.4 & 3 & MR & 7.3 & 3 & MR & 9 & MR \\
\hline $\mathbf{2 1 5}$ & C-1548 & 11.0 & 5 & MS & 8.6 & 3 & MR & 10 & MR \\
\hline Checks & TN1 & 22.7 & 7 & S & 17.6 & 7 & S & 20 & S \\
\cline { 2 - 13 } & Local & 17.0 & 7 & S & 14.6 & 5 & MS & 16 & S \\
\hline \multicolumn{2}{|c|}{ Mean } & 11.47 & - & - & 9.98 & - & - & 10.73 & - \\
\hline \multicolumn{2}{|c|}{ Std. Dev. } & 7.26 & - & - & 7.58 & - & - & 7.42 & - \\
\hline \multicolumn{2}{|c|}{ Std. Error } & 0.49 & - & - & 0.51 & - & - & 0.50 & - \\
\hline \multicolumn{2}{|c|}{ CV(\%) } & 63.30 & - & - & 75.86 & - & - & 69.58 & - \\
\hline
\end{tabular}

Table.5 The promising rice genotypes identified against rice yellow stem borer

\begin{tabular}{|c|c|c|c|c|c|c|c|c|c|c|c|c|c|}
\hline \multirow[t]{3}{*}{ S.No } & \multirow[t]{3}{*}{ IC No. } & \multicolumn{6}{|c|}{ Dead hearts } & \multicolumn{6}{|c|}{ White ears } \\
\hline & & \multicolumn{2}{|c|}{ kharif 2016} & \multicolumn{2}{|c|}{ kharif 2017} & \multicolumn{2}{|c|}{ Mean } & \multicolumn{2}{|c|}{ kharif 2016} & \multicolumn{2}{|c|}{ kharif 2017} & \multicolumn{2}{|c|}{ Mean } \\
\hline & & $\begin{array}{c}\% \\
\text { DH }\end{array}$ & Status & $\begin{array}{c}\% \\
\text { DH }\end{array}$ & Status & $\begin{array}{c}\% \\
\text { DH }\end{array}$ & Status & $\begin{array}{c}\% \\
\text { WE }\end{array}$ & Status & $\begin{array}{r}\% \\
\text { WE }\end{array}$ & Status & $\begin{array}{c}\% \\
\text { WE }\end{array}$ & Status \\
\hline 1 & 381538 & 11.6 & MR & 14.3 & MR & 13 & MR & 10.8 & MS & 10.1 & MR & 10 & MR \\
\hline 2 & 450535 & 16.9 & MR & 11.4 & MR & 14 & MR & 6.5 & MR & 3.5 & $\mathrm{R}$ & 5 & $\mathrm{R}$ \\
\hline 3 & 463380 & 10.5 & MR & 10.8 & MR & 11 & MR & 4.3 & $\mathrm{R}$ & 2.9 & $\mathrm{R}$ & 4 & $\mathrm{R}$ \\
\hline 4 & 464140 & 16.9 & MR & 13.4 & MR & 15 & MR & 5.6 & MR & 5.0 & $\mathrm{R}$ & 5 & $\mathrm{R}$ \\
\hline 5 & 464186 & 16.8 & MR & 13.4 & MR & 15 & MR & 4.3 & $\mathrm{R}$ & 4.0 & $\mathrm{R}$ & 4 & $\mathrm{R}$ \\
\hline 6 & 574807 & 16.3 & MR & 13.2 & MR & 15 & MR & 6.1 & MR & 4.1 & $\mathrm{R}$ & 5 & $\mathrm{R}$ \\
\hline 7 & 578388 & 11.9 & MR & 10.8 & MR & 11 & MR & 4.0 & $\mathrm{R}$ & 3.2 & $\mathrm{R}$ & 4 & $\mathrm{R}$ \\
\hline
\end{tabular}

MR : Moderately Resistant; R : Resistant

Identification of resistant entries of rice genotypes against yellow stem borer (pooled mean of two seasons)

The cumulative mean of per cent white ear damage by yellow stem borer for two consecutive kharif seasons inferred that out of 215 rice genotypes screened at field level, 14 entries registered stem borer incidence less than 5.0 per cent and categorized as resistant entries (R). A sum of 101, 82 and 17 entries were rated as moderately resistant (MR), moderately susceptible (MS) and susceptible (S) with per cent white ears damage ranged from 6-10, 11-15 and 16-20 per cent, respectively. One entry (C-490) had exhibited highly susceptible (HS) status with 30 per 
cent WE as against the lowest in C-1433 and C-1464 entries with only 3.0 per cent white ears damage.

Considering the influence of damage by yellow stem borer at vegetative stage and reproductive stage in terms of dead hearts and white ears, respectively and their impact on yield only seven genotypes were identified as resistance/ moderately resistant entries out of 215 entries screened, that had exhibited lowest pest damage and considered as promising entries to be utilized for further biochemical analysis and breeding programmes. The selected seven promising genotypes of rice with indigenous collection number (IC no) were 381538, 450535, 463380, 464140, 464186, 574807 and 578388 , respectively (Table 5).

From the present investigation trial, it was clearly witnessed that, most of the rice genotypes which were resistance/ moderately resistant to the dead hearts damage by yellow stem borer at vegetative stage had exhibited moderately susceptible or susceptible reaction to white ears damage at reproductive stage and vice versa. The result was in agreement with the findings of Pathak et al., (1971) who inferred that the rice varieties exhibiting resistance at dead heart stage were found susceptible at white ear stage demonstrating the resistance at both stages as independent factors.

The screening studies by Pandey and Choubey (2011) also supported the present results stating that resistance reaction by rice varieties against yellow stem borer differed among the seasons. They have screened 60 rice germplasm against rice stem borer for two successive kharif seasons (2003 \& 2004) and notified that among 60 rice entries 35, 29 and 6 entries were rated as resistant, moderately resistant and susceptible respectively. Whereas 31,21 and 8 germplasm were rated as resistant, moderately resistant and susceptible entries, respectively during kharif, 2004.

The above reports indicated variation in performance of rice varieties among seasons and were further supported by findings of Justin and Preetha (2014) who screened 77 genotypes during kharif, 2011 \& 2012 and 57 genotypes during rabi 2011 \& 2012 for their reaction to rice YSB. During kharif 2011, the genotypes TP 08079, TP 10015, TP 10019, TP 10029 and TP 10031 were promising with meager incidence and rated as highly resistant. While, the genotypes TP 10006, TP 10007, TP 10008, TP 10009, TP 10010, TP10011 and TP 10012 were highly resistant with scale ' 0 ' at both vegetative and reproductive stages of kharif, 2012. Out of 57 genotypes screened for the year, rabi 2011, TP 10007 recorded nil incidence and rated as highly resistant while at rabi 2012, 15 genotypes recorded nil incidence of stem borer. The genotype TP 10052 \& TP 08033 and T P 09119 were rated as resistant during kharif and rabi seasons, respectively.

Similar screening trials in rice by Mohan et al., (2003) stated that W1263 and TKM 6 had exhibited significantly low damage of YSB infesting rice at both vegetative $(\% \mathrm{DH})$ and reproductive stages (\% WE) of crop growth. Prasad et al., (2015) evaluated 55 promising rice genotypes against yellow stemborer along with susceptible check variety (TN1) and resistant check (variety Suraksha) and inferred that genotypes RP-Bio-Patho-02, BPT-5204 and R-DRR-02 were promising and highly resistant with $0.67,0.78$ and 1.22 per cent mean stem borer infestation in comparison to susceptible check and resistant check with 20.69 and 5.22 per cent mean YSB infestation, respectively. Field screening trails by Rishikesh et al., (2018) for 73 rice genotypes during kharif 2016 and 2017 against rice yellow stem borer witnessed that lowest white ears damage was recorded in IR 36, R 1700-302-1-156-1, Shyamla and IR 64 
with $0.0,0.17,0.17 \& 0.1$ per cent white ears per plant on each genotypes, respectively

In conclusions, field screening trials revealed seven promising rice genotypes (IC No. 381538, 450535, 463380, 464140, 464186, 574807 and 578388) exhibiting moderate resistance/ resistance to yellow stem borer both in terms of per cent dead hearts and white ears. The selected promising rice germplasm against yellow stem borer from the present investigations can be further studied for bio chemical analysis. The molecular characterization and identification of QTLs for resistance against stem borers through molecular markers may be utilized for introgression of resistant genes in the breeding programmes of rice cultures.

\section{Acknowledgements}

The authors express our heartfelt thanks to Dr. L.V. Subba Rao, Principal Scientist, Indian Institute of Rice Research, Hyderabad towards sparing the rice accessions necessary for the above research.

\section{References}

Agricultural Statistics at a glance (2016) Government of India Ministry of Agriculture \& Farmers Welfare Department of Agriculture, Cooperation \& Farmers Welfare Directorate of Economics and Statistic. https://eands.dacnet.nic.in.

Justin, C and Preetha, G. 2014. Screening of rice cultures/ genotypes for their reaction to yellow stem borer, Scirpophaga incertulas
Walker. Research Journal of Agriculture and Environmental Management. 3 (12): 646-652.

Mohan, K.S., Thiruvengadam, V., Samiayyan K and Shanmugasundaram, P. 2003. Generation and screening of recombinant inbred lines of rice for yellow stem borer resistance. Indian Journal of Experimental Biology. 41: 346-351.

Pandey, S and Choubey, M.N. 2011. Screening of different rice germplasm for resistance to yellow stem borer, Scirpophaga incertulas (Walker). Agricultural Science Digest. 31 (2):140-143.

Pasalu, I.C and Katti, G. 2006. Advances in ecofriendly approaches in rice IPM. Journal of Rice Research. 1 (1): 83-90.

Pathak, M.D., Andres, F., Galacajac, N and Romas, R. 1971. Resistance of rice varieties to striped stem borer. International Rice Research institute. Technical Bulletin. 11: 69.

Prasad S.S., Gupta P.K., Singh R.V and Mishra J.P. 2015. Identification of rice donors resistant against yellow stem borer, Scirpophaga incertulas (Walker). Scholars Journal of Agriculture and Veterinary Sciences. 2 (1A): 24-26.

Prasad, S.S., Gupta, P.K and Kanaujia, B.L. 2007. Simulation study on yield loss due to Scirpophaga incertulas on semi deep water rice. Annuals of Plant Protection Sciences. 15: 491-492.

Rishikesh Mandloi, A., Shukla, T., Venkatesan, A.K., Bhowmick and SK Singh. 2018. Screening of rice (Oryza sativa L.) varieties and genotypes against Scirpophaga incertulas (Walker). Journal of Entomology and Zoology Studies. 6 (3): 1195-1199.

\section{How to cite this article:}

Sudha Rani, D., Ch. Chiranjeevi, T. Madhumathi, S. Krishnam Raju and Nafeez Umar, Sk. 2020. Identification of Rice Genotypes for Resistance against Yellow Stem Borer in Irrigated Rice. Int.J.Curr.Microbiol.App.Sci. 9(05): 1627-1643.

doi: https://doi.org/10.20546/ijcmas.2020.905.184 